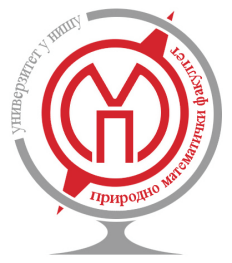

\title{
Fixed Point Results for F-Contractive Mappings of Hardy-Rogers-Type
}

\author{
Monica Cosentino, Pasquale Vetro ${ }^{a}$ \\ ${ }^{a}$ Università degli Studi di Palermo, Dipartimento di Matematica e Informatica, Via Archirafi, 34, 90123 Palermo, Italy
}

\begin{abstract}
Recently, Wardowski introduced a new concept of contraction and proved a fixed point theorem which generalizes Banach contraction principle. Following this direction of research, in this paper, we will present some fixed point results of Hardy-Rogers-type for self-mappings on complete metric spaces or complete ordered metric spaces. Moreover, an example is given to illustrate the usability of the obtained results.
\end{abstract}

\section{Introduction}

It is well known that the contraction mapping principle, formulated and proved in the Ph.D. dissertation of Banach in 1920, which was published in 1922 [4], is one of the most important theorems in classical functional analysis. Indeed it is widely considered as the source of metric fixed point theory. Also its significance lies in its vast applicability in a number of branches of mathematics. Starting from these considerations, the study of fixed and common fixed points of mappings satisfying a certain metrical contractive condition attracted many researchers, see for example [1-3, 5, 6, 8-11, 16, 22-27]. The reader can also see [18, 20,21], for existence results of fixed points for contractive non-self-mappings.

Recently, Wardowski [28] introduced a new concept of contraction and proved a fixed point theorem which generalizes Banach contraction principle. Following this direction of research, in this paper, we will present some fixed point results of Hardy-Rogers-type for self-mappings on complete metric spaces or complete ordered metric spaces. Moreover, an example is given to illustrate the usability of the obtained results.

\section{Preliminaries}

The aim of this section is to present some notions and results used in the paper. Throughout the article we denote by $\mathbb{R}$ the set of all real numbers, by $\mathbb{R}^{+}$the set of all positive real numbers and by $\mathbb{N}$ the set of all positive integers.

Definition 2.1. Let $F: \mathbb{R}^{+} \rightarrow \mathbb{R}$ be a mapping satisfying:

(F1) $F$ is strictly increasing;

2010 Mathematics Subject Classification. Primary 47H10; Secondary 54H25

Keywords. Metric spaces, ordered metric spaces, fixed points, F-contractions of Hardy-Rogers-type.

Received: 27 September 2013; Accepted: 02 November 2013

Communicated by Vladimir Rakočević

Research supported by Università degli Studi di Palermo, Local Project R.S. ex 60\%.

Email addresses: mcosentino1@alice.it (Monica Cosentino), vetro@math.unipa.it (Pasquale Vetro) 
(F2) for each sequence $\left\{\alpha_{n}\right\} \subset \mathbb{R}^{+}$of positive numbers $\lim _{n \rightarrow+\infty} \alpha_{n}=0$ if and only if $\lim _{n \rightarrow+\infty} F\left(\alpha_{n}\right)=-\infty$;

(F3) there exists $k \in(0,1)$ such that $\lim _{\alpha \rightarrow 0^{+}} \alpha^{k} F(\alpha)=0$.

We denote with $\mathcal{F}$ the family of all functions $F$ that satisfy the conditions (F1)-(F3).

Definition 2.2 ([28]). Let $(X, d)$ be a metric space. A self-mapping $T$ on $X$ is called an F-contraction if there exist $F \in \mathcal{F}$ and $\tau \in \mathbb{R}^{+}$such that

$$
\tau+F(d(T x, T y)) \leq F(d(x, y))
$$

for all $x, y \in X$ with $d(T x, T y)>0$.

Definition 2.3. Let $(X, d)$ be a metric space. A self-mapping $T$ on $X$ is called an F-contraction of Hardy-Rogers-type if there exist $F \in \mathcal{F}$ and $\tau \in \mathbb{R}^{+}$such that

$$
\tau+F(d(T x, T y)) \leq F(\alpha d(x, y)+\beta d(x, T x)+\gamma d(y, T y)+\delta d(x, T y)+L d(y, T x)),
$$

for all $x, y \in X$ with $d(T x, T y)>0$, where $\alpha+\beta+\gamma+2 \delta=1, \gamma \neq 1$ and $L \geq 0$.

By choosing opportunely the mapping $F$, we obtain certain classes of contractions known in the literature, as shown with the following examples.

Example 2.4 ([28]). Let $F: \mathbb{R}^{+} \rightarrow \mathbb{R}$ be given by $F(x)=\ln x$. It is clear that $F$ satisfies (F1)-(F2) and (F3) for any $k \in(0,1)$. Each mapping $T: X \rightarrow X$ satisfying $(1)$ is an F-contraction such that

$$
d(T x, T y) \leq e^{-\tau} d(x, y), \quad \text { for all } x, y \in X, T x \neq T y .
$$

It is clear that for $x, y \in X$ such that $T x=T y$ the previous inequality also holds and hence $T$ is a contraction.

Example 2.5 ([28]). Let $F: \mathbb{R}^{+} \rightarrow \mathbb{R}$ be given by $F(x)=\ln x+x$. It is clear that $F$ satisfies (F1)-(F3). Each mapping $T: X \rightarrow X$ satisfying (1) is an F-contraction such that

$$
\frac{d(T x, T y)}{d(x, y)} e^{d(T x, T y)-d(x, y)} \leq e^{-\tau}, \quad \text { for all } x, y \in X, T x \neq T y .
$$

Remark 2.6. From (F1) and (1), we deduce that every F-contraction $T$ is a contractive mapping, that is,

$$
d(T x, T y)<d(x, y), \quad \text { for all } x, y \in X, T x \neq T y .
$$

From (F1) and (2), we deduce that every F-contraction of Hardy-Rogers-type T satisfies the following condition:

$$
d(T x, T y))<\alpha d(x, y)+\beta d(x, T x)+\gamma d(y, T y)+\delta d(x, T y)+L d(y, T x),
$$

for all $x, y \in X, T x \neq T y$, where $\alpha+\beta+\gamma+2 \delta=1, \gamma \neq 1$ and $L \geq 0$.

Let $X$ be a non-empty set. If $(X, d)$ is a metric space and $(X, \leq)$ is partially ordered, then $(X, d, \leq)$ is called an ordered metric space. Then $x, y \in X$ are called comparable if $x \leq y$ or $y \leq x$ holds. Let $(X, \leq)$ be a partially ordered set. A self-mapping $T$ on $X$ is called non-decreasing if $T x \leq T y$ whenever $x \leq y$ for all $x \in X$. An ordered metric space $(X, d, \leq)$ is regular if

(r) for every non-decreasing sequence $\left\{x_{n}\right\}$ in $X$ convergent to some $x \in X$, we have $x_{n} \leq x$ for all $n \in \mathbb{N} \cup\{0\}$. 


\section{Fixed points for F-contractions of Hardy-Rogers-type}

In this section, we give some fixed point results for F-contractions of Hardy-Rogers-type in a complete metric space.

Theorem 3.1. Let $(X, d)$ be a complete metric space and let $T$ be a self-mapping on $X$. Assume that there exist $F \in \mathcal{F}$ and $\tau \in \mathbb{R}^{+}$such that $T$ is an F-contraction of Hardy-Rogers-type, that is,

$$
\tau+F(d(T x, T y)) \leq F(\alpha d(x, y)+\beta d(x, T x)+\gamma d(y, T y)+\delta d(x, T y)+L d(y, T x)),
$$

for all $x, y \in X, T x \neq T y$, where $\alpha+\beta+\gamma+2 \delta=1, \gamma \neq 1$ and $L \geq 0$. Then $T$ has a fixed point. Moreover, if $\alpha+\delta+L \leq 1$, then the fixed point of $T$ is unique.

Proof. Let $x_{0} \in X$ be an arbitrary point, and let $\left\{x_{n}\right\}$ be the Picard sequence with initial point $x_{0}$, that is, $x_{n}=T^{n} x_{0}=T x_{n-1}$. If $x_{n}=x_{n-1}$ for some $n \in \mathbb{N}$, then $x_{n}$ is a fixed point of $T$. Now, let $d_{n}=d\left(x_{n}, x_{n+1}\right)$ for all $n \in \mathbb{N} \cup\{0\}$. If $x_{n} \neq x_{n+1}$, that is, $T x_{n-1} \neq T x_{n}$ for all $n \in \mathbb{N}$, using the contractive condition (4) with $x=x_{n-1}$ and $y=x_{n}$, we get

$$
\begin{aligned}
\tau+F\left(d_{n}\right) & =\tau+F\left(d\left(x_{n}, x_{n+1}\right)\right)=\tau+F\left(d\left(T x_{n-1}, T x_{n}\right)\right) \\
& \leq F\left(\alpha d\left(x_{n-1}, x_{n}\right)+\beta d\left(x_{n-1}, T x_{n-1}\right)+\gamma d\left(x_{n}, T x_{n}\right)+\delta d\left(x_{n-1}, T x_{n}\right)+\operatorname{Ld}\left(x_{n}, T x_{n-1}\right)\right) \\
& =F\left(\alpha d\left(x_{n-1}, x_{n}\right)+\beta d\left(x_{n-1}, x_{n}\right)+\gamma d\left(x_{n}, x_{n+1}\right)+\delta d\left(x_{n-1}, x_{n+1}\right)+\operatorname{Ld}\left(x_{n}, x_{n}\right)\right) \\
& =F\left(\alpha d_{n-1}+\beta d_{n-1}+\gamma d_{n}+\delta d\left(x_{n-1}, x_{n+1}\right)\right) \\
& \leq F\left((\alpha+\beta) d_{n-1}+\gamma d_{n}+\delta\left[d_{n-1}+d_{n}\right]\right) \\
& =F\left((\alpha+\beta+\delta) d_{n-1}+(\gamma+\delta) d_{n}\right) .
\end{aligned}
$$

Since $F$ is strictly increasing, we deduce

$$
d_{n}<(\alpha+\beta+\delta) d_{n-1}+(\gamma+\delta) d_{n}
$$

and hence

$$
(1-\gamma-\delta) d_{n}<(\alpha+\beta+\delta) d_{n-1}, \quad \text { for all } n \in \mathbb{N} .
$$

From $\alpha+\beta+\gamma+2 \delta=1$ and $\gamma \neq 1$, we deduce that $1-\gamma-\delta>0$ and so

$$
d_{n}<\frac{\alpha+\beta+\delta}{1-\gamma-\delta} d_{n-1}=d_{n-1}, \quad \text { for all } n \in \mathbb{N} .
$$

Consequently,

$$
\tau+F\left(d_{n}\right) \leq F\left(d_{n-1}\right), \quad \text { for all } n \in \mathbb{N} .
$$

This implies

$$
F\left(d_{n}\right) \leq F\left(d_{n-1}\right)-\tau \leq \cdots \leq F\left(d_{0}\right)-n \tau, \quad \text { for all } n \in \mathbb{N}
$$

and so $\lim _{n \rightarrow+\infty} F\left(d_{n}\right)=-\infty$. By the properties (F2), we get that $d_{n} \rightarrow 0$ as $n \rightarrow+\infty$.

Now, let $k \in(0,1)$ such that $\lim _{n \rightarrow+\infty} d_{n}^{k} F\left(d_{n}\right)=0$. By (5), the following holds for all $n \in \mathbb{N}$ :

$$
d_{n}^{k} F\left(d_{n}\right)-d_{n}^{k} F\left(d_{0}\right) \leq d_{n}^{k}\left(F\left(d_{0}\right)-n \tau\right)-d_{n}^{k} F\left(d_{0}\right)=-n \tau d_{n}^{k} \leq 0 .
$$

Letting $n \rightarrow+\infty$ in (6), we deduce $\lim _{n \rightarrow+\infty} n d_{n}^{k}=0$ and hence $\lim _{n \rightarrow+\infty} n^{1 / k} d_{n}=0$. Now, $\lim _{n \rightarrow+\infty} n^{1 / k} d_{n}=0$ ensures that the series $\sum_{n=1}^{+\infty} d_{n}$ is convergent. This implies that $\left\{x_{n}\right\}$ is a Cauchy sequence. As $X$ is a complete metric space there exists $z \in X$ such that $x_{n} \rightarrow z$. If $z=T z$ the proof is finished. Assume that $z \neq T z$. If $T x_{n}=T z$ for infinite values of $n \in \mathbb{N} \cup\{0\}$, then the sequence $\left\{x_{n}\right\}$ has a subsequence that converges to $T z$ 
and the uniqueness of the limit implies $z=T z$. Then we can assume that $T x_{n} \neq T z$ for all $n \in \mathbb{N} \cup\{0\}$. Now, by (3), we have

$$
\begin{aligned}
d(z, T z) & \leq d\left(z, x_{n+1}\right)+d\left(T x_{n}, T z\right) \\
& <d\left(z, x_{n+1}\right)+\alpha d\left(x_{n}, z\right)+\beta d\left(x_{n}, T x_{n}\right)+\gamma d(z, T z)+\delta d\left(x_{n}, T z\right)+L d\left(z, T x_{n}\right) \\
& =d\left(z, x_{n+1}\right)+\alpha d\left(x_{n}, z\right)+\beta d\left(x_{n}, x_{n+1}\right)+\gamma d(z, T z)+\delta d\left(x_{n}, T z\right)+L d\left(z, x_{n+1}\right) .
\end{aligned}
$$

Letting $n \rightarrow+\infty$ in the previous inequality, we get

$$
d(z, T z) \leq(\gamma+\delta) d(z, T z)<d(z, T z)
$$

which is a contradiction and hence $z=T z$.

Now, we prove the uniqueness of the fixed point. Assume that $w \in X$ is another fixed point of $T$, different from $z$. This means that $d(z, w)>0$. Taking $x=z$ and $y=w$ in (4), we have

$$
\begin{aligned}
\tau+F(d(z, w)) & =\tau+F(d(T z, T w)) \\
& \leq F(\alpha d(z, w)+\beta d(z, T z)+\gamma d(w, T w)+\delta d(z, T w)+L d(w, T z)) \\
& =F((\alpha+\delta+L) d(z, w)),
\end{aligned}
$$

which is a contradiction, if $\alpha+\delta+L \leq 1$, and hence $z=w$.

As a first corollary of Theorem 3.1, taking $\alpha=1$ and $\beta=\gamma=\delta=L=0$, we obtain Theorem 2.1 of Wardowski [28]. Further, putting $\alpha=\delta=L=0$ and $\beta+\gamma=1$ and $\beta \neq 0$, we obtain the following version of Kannan's result [12].

Corollary 3.2. Let $(X, d)$ be a complete metric space and let $T$ be a self-mapping on $X$. Assume that there exist $F \in \mathcal{F}$ and $\tau \in \mathbb{R}^{+}$such that

$$
\tau+F(d(T x, T y)) \leq F(\beta d(x, T x)+\gamma d(y, T y)),
$$

for all $x, y \in X, T x \neq T y$, where $\beta+\gamma=1, \gamma \neq 1$. Then $T$ has a unique fixed point in $X$.

A version of the Chatterjea [7] fixed point theorem is obtained from the Theorem 3.1 putting $\alpha=\beta=\gamma=0$ and $\delta=1 / 2$.

Corollary 3.3. Let $(X, d)$ be a complete metric space and let $T$ be a self-mapping on $X$. Assume that there exist $F \in \mathcal{F}$ and $\tau \in \mathbb{R}^{+}$such that

$$
\tau+F(d(T x, T y)) \leq F\left(\frac{1}{2} d(x, T y)+L d(y, T x)\right),
$$

for all $x, y \in X, T x \neq T y$. Then $T$ has a fixed point in $X$. If $L \leq 1 / 2$, then the fixed point of $T$ is unique.

Finally, if we choose $\delta=L=0$, we obtain a Reich [19] type theorem.

Corollary 3.4. Let $(X, d)$ be a complete metric space and let $T$ be a self-mapping on $X$. Assume that there exist $F \in \mathcal{F}$ and $\tau \in \mathbb{R}^{+}$such that

$$
\tau+F(d(T x, T y)) \leq F(\alpha d(x, y)+\beta d(x, T x)+\gamma d(y, T y)),
$$

for all $x, y \in X, T x \neq T y$, where $\alpha+\beta+\gamma=1, \gamma \neq 1$. Then $T$ has a unique fixed point in $X$. 


\section{Fixed points in ordered metric spaces}

The existence of fixed points of self-mappings defined on certain type of ordered sets plays an important role in the order theoretic approach. It has been initiated in 2004 by Ran and Reurings [17], and further studied by Nieto and Rodríguez-Lopez [13]. Then, several interesting and valuable results have appeared in this direction $[1,14-16,24]$.

Theorem 4.1. Let $(X, d, \leq)$ be an ordered complete metric space and let $T$ be a non-decreasing self-mapping on $X$. Assume that there exist $F \in \mathcal{F}$ and $\tau \in \mathbb{R}^{+}$such that $T$ is an ordered F-contraction of Hardy-Rogers-type, that is,

$$
\tau+F(d(T x, T y)) \leq F(\alpha d(x, y)+\beta d(x, T x)+\gamma d(y, T y)+\delta d(x, T y)+L d(y, T x)),
$$

for all comparable $x, y \in X, T x \neq T y$, where $\alpha+\beta+\gamma+2 \delta=1, \gamma \neq 1$ and $L \geq 0$. If the following conditions are satisfied:

(i) there exists $x_{0} \in X$ such that $x_{0} \leq T x_{0}$;

(ii) $X$ is regular;

then $T$ has a fixed point. Moreover, if $\alpha+\delta+L \leq 1$, then the set of fixed points of $T$ is well ordered if and only if $T$ has a unique fixed point.

Proof. Let $x_{0} \in X$ be such that $x_{0} \leq T x_{0}$, and let $\left\{x_{n}\right\}$ be the Picard sequence of initial point $x_{0}$, that is, $x_{n}=T^{n} x_{0}=T x_{n-1}$. If $x_{n}=x_{n-1}$ for some $n \in \mathbb{N}$, then $x_{n}$ is a fixed point of $T$. Now, let $d_{n}=d\left(x_{n}, x_{n+1}\right)$ for all $n \in \mathbb{N} \cup\{0\}$. Assume that $x_{n} \neq x_{n-1}$ for all $n \in \mathbb{N}$. As $T$ is non-decreasing and $x_{0} \leq T x_{0}$, we deduce that

$$
x_{0}<x_{1}<\cdots<x_{n}<\cdots,
$$

that is, $x_{n}$ and $x_{n+1}$ are comparable and $T x_{n-1} \neq T x_{n}$ for all $n \in \mathbb{N} \cup 0$.

Proceeding as in the proof of Theorem 3.1, we obtain that $\left\{x_{n}\right\}$ is a Cauchy sequence. As $X$ is a complete metric space there exists $z \in X$ such that $x_{n} \rightarrow z$. If $z=T z$ the proof is finished. Assume that $z \neq T z$. Since $X$ is regular, from (8), we deduce that $x_{n}$ and $z$ are comparable and $T x_{n} \neq T z$ for all $n \in \mathbb{N} \cup\{0\}$.

Now, using (3), we obtain

$$
\begin{aligned}
d(z, T z) & \leq d\left(z, x_{n+1}\right)+d\left(T x_{n}, T z\right) \\
& <d\left(z, x_{n+1}\right)+\alpha d\left(x_{n}, z\right)+\beta d\left(x_{n}, x_{n+1}\right)+\gamma d(z, T z)+\delta d\left(x_{n}, T z\right)+L d\left(z, x_{n+1}\right) .
\end{aligned}
$$

Letting $n \rightarrow+\infty$ in the previous inequality, we get

$$
d(z, T z) \leq(\gamma+\delta) d(z, T z)<d(z, T z),
$$

which is a contradiction and hence $z=T z$.

Now, we assume that $\alpha+\delta+L \leq 1$ and that the set of fixed points of $T$ is well ordered. We claim that the fixed point of $T$ is unique. Assume on the contrary that there exists another fixed point $w$ in $X$ such that $z \neq w$. Using the condition (7), with $x=z$ and $y=w$, we get

$$
\begin{aligned}
\tau+F(d(z, w)) & =\tau+F(d(T z, T w)) \\
& \leq F(\alpha d(z, w)+\beta d(z, T z)+\gamma d(w, T w)+\delta d(z, T w)+L d(w, T z)) \\
& =F((\alpha+\delta+L) d(z, w)) \\
& \leq F(d(z, w)),
\end{aligned}
$$

which is a contradiction and hence $z=w$. Conversely, if $T$ has a unique fixed point, then the set of fixed points of $T$, being a singleton, is well ordered.

Theorem 4.2. Let $(X, d, \leq)$ be an ordered complete metric space and let $T$ be a non-decreasing self-mapping on $X$. Assume that there exist $F \in \mathcal{F}$ and $\tau \in \mathbb{R}^{+}$such that $T$ is an ordered F-contraction of Hardy-Rogers-type. If the following conditions are satisfied: 
(i) there exists $x_{0} \in X$ such that $x_{0} \leq T x_{0}$;

(ii) $\mathrm{X}$ is regular;

then $T$ has a fixed point. Moreover, if $\alpha+2 \gamma+\delta+L<1$ and the following condition holds:

(iii) for all $z, w \in X$ there exists $v \in X$ such that $z$ and $v$ are comparable and $w$ and $v$ are comparable;

then $T$ has a unique fixed point.

Proof. The existence of a fixed point of $T$ is consequence of Theorem 4.1. Now, let $z \in X$ be a fixed point of $T$. For all $v \in X$ comparable with $z$ such that $T z \neq T v$, we have

$$
\begin{aligned}
\tau+F(d(T z, T v)) & \leq F(\alpha d(z, v)+\beta d(z, T z)+\gamma d(v, T v)+\delta d(z, T v)+L d(v, T z)) \\
& \leq F(\alpha d(z, v)+\gamma(d(v, z)+d(z, T v))+\delta d(z, T v)+L d(v, z)) \\
& =F((\alpha+\gamma+L) d(z, v)+(\gamma+\delta) d(z, T v)) .
\end{aligned}
$$

Since $F$ is strictly increasing, we deduce

$$
d(z, T v)<(\alpha+\gamma+L) d(z, v)+(\gamma+\delta) d(z, T v)
$$

and hence

$$
(1-\gamma-\delta) d(z, T v)<(\alpha+\gamma+L) d(z, v)
$$

Since $1-\gamma-\delta>0$, we get

$$
d(z, T v)<\frac{\alpha+\gamma+L}{1-\gamma-\delta} d(z, v) .
$$

As $T$ is non-decreasing we have that $z$ and $T^{n} v$ are comparable for all $n \in \mathbb{N}$. If $z \neq T^{n} v$ for all $n \in \mathbb{N}$, then

$$
d\left(z, T^{n} v\right)<\lambda^{n} d(z, v), \quad \text { for all } n \in \mathbb{N},
$$

where $\lambda=\frac{\alpha+\gamma+L}{1-\gamma-\delta}<1$. From the previous inequality, we obtain $d\left(z, T^{n} v\right) \rightarrow 0$ as $n \rightarrow+\infty$.

Now, if $z, w$ are two fixed points of $T$, by the condition (iii) there exists $v \in X$ such that $z$ and $v$ are comparable and $w$ and $v$ are comparable. If $z=T^{n} v$ or $w=T^{n} v$ for some $n \in \mathbb{N}$, then $z$ and $w$ are comparable and the uniqueness of the fixed point follows since $T$ is an F-contraction of Hardy-Rogers-type. Assume that $z \neq T^{n} v$ and $w \neq T^{n} v$ for all $n \in \mathbb{N}$. Then

$$
d(z, w) \leq d\left(z, T^{n} v\right)+d\left(w, T^{n} v\right) \rightarrow 0, \quad \text { as } n \rightarrow+\infty
$$

and hence $d(z, w)=0$, that is, $z=w$.

If in Theorems 4.1 and 4.2 , we choose opportunely the mapping $F$, then we obtain some results of fixed point in the setting of ordered metric spaces known in the literature. For example, if we choose $F(x)=\ln x$ in Theorem 4.1 and putting $\beta=\gamma=\delta=L=0$ and $\tau=1$, then we obtain Theorem 2.2 of [13].

Example 4.3. Let $X=\left\{S_{n}:=\frac{n(n+1)}{2}: n \in \mathbb{N}\right\}$ and $d(x, y)=|x-y|$ for all $x, y \in X$. Clearly, $(X, d)$ is a complete metric space. Let $T$ be the self-mapping on $X$ defined by

$$
T S_{n}= \begin{cases}S_{1} & \text { if } n=1, \\ S_{n+1} & \text { if } n \text { is even, } \\ S_{n-1} & \text { if } n>1 \text { is odd } .\end{cases}
$$


Firstly observe that $T S_{m} \neq T S_{n}$ for $m \neq n$. Also the mapping $T$ is not an F-contraction if we choose $F(x)=x+\ln x$. In fact, for every $m, n \in \mathbb{N}$ with $m>n$, we have

$$
\begin{aligned}
\frac{d\left(T S_{2 m}, T S_{2 n}\right)}{d\left(S_{2 m}, S_{2 n}\right)} e^{\left.d\left(T S_{2 m}, T S_{2 n}\right)\right)-d\left(S_{2 m}, S_{2 n}\right)} & =\frac{S_{2 m+1}-S_{2 n+1}}{S_{2 m}-S_{2 n}} e^{S_{2 m+1}-S_{2 n+1}-S_{2 m}+S_{2 n}} \\
& =\frac{2(m+n)+3}{2(m+n)+1} e^{2(m-n)} \\
& \geq 1>e^{-\tau},
\end{aligned}
$$

for all $\tau \in \mathbb{R}^{+}$. This implies that $T$ is not an F-contraction (see Example 2.5).

Now, we consider the partial order $\leq$ on $X$ defined by

$$
S_{m} \leq S_{n} \quad \text { if } \quad(m=n) \quad \text { or } \quad(m<n \text { and } m, n \text { odd }) .
$$

We show that $T$ is an ordered F-contraction of Hardy-Rogers-type with $\beta=\gamma=\delta=L=0$ and $\tau=1$. To see this, for $m>n>1$ and $m, n$ odd, let us consider the following calculations:

$$
\begin{aligned}
\frac{d\left(T S_{m}, T S_{n}\right)}{d\left(S_{m}, S_{n}\right)} e^{d\left(T S_{m}, T S_{n}\right)-d\left(S_{m}, S_{n}\right)} & =\frac{S_{m-1}-S_{n-1}}{S_{m}-S_{n}} e^{S_{m-1}-S_{n-1}-S_{m}+S_{n}} \\
& =\frac{m+n-1}{m+n+1} e^{n-m} \\
& \leq e^{-1}
\end{aligned}
$$

Obviously, the same holds if $m>n=1$. Next, if $\left\{x_{n}\right\}$ is a convergent non-decreasing sequence, then there is $m \in \mathbb{N}$ such that $x_{n}=x_{m}$ for all $n \geq m$ and so $X$ is regular. As $S_{1} \leq T S_{1}$, all the conditions of Theorem 4.1 are satisfied and hence $T$ has a fixed point. Clearly, $S_{1}$ is a fixed point of $T$.

\section{References}

[1] I. Altun, G. Durmaz, Some fixed point theorems on ordered cone metric spaces, Rendiconti del Circolo Matematico di Palermo 58 (2009) 319-325.

[2] M. Arshad, A. Azam, P. Vetro, Some common fixed point results in cone metric spaces, Fixed Point Theory and Applications 2009 (2009) 11 pp., Article ID 493965.

[3] A. Azam, M. Arshad, I. Beg, Common fixed points of two maps in cone metric spaces, Rendiconti del Circolo Matematico di Palermo 57 (2008) 433-441.

[4] S. Banach, Sur les opérations dans les ensembles abstraits et leur application aux équations intégrales, Fundamenta Mathematicae 3 (1922) 133-181.

[5] V. Berinde, F. Vetro, Common fixed points of mappings satisfying implicit contractive conditions, Fixed Point Theory and Applications 2012:105 (2012).

[6] V. Berinde, F. Vetro, Fixed point for cyclic weak $(\psi, C)$-contractions in 0-complete partial metric spaces, Filomat 27 (2013) $1405-$ 1413.

[7] S.K. Chatterjea, Fixed-point theorems, Comptes Rendus de l'Academie Bulgare des Sciences 25 (1972) 727-730.

[8] C. Di Bari, P. Vetro, $\varphi$-pairs and common fixed points in cone metric spaces, Rendiconti del Circolo Matematico di Palermo 57 (2008) 279-285.

[9] C. Di Bari, P. Vetro, Weakly $\varphi$-pairs and common fixed points in cone metric spaces, Rendiconti del Circolo Matematico di Palermo 58 (2009) 125-132.

[10] M. Edelstein, On fixed and periodic points under contractive mappings, Journal of the London Mathematical Society 37 (1962) 74-79.

[11] L.-G. Huang, X. Zhang, Cone metric spaces and fixed point theorems of contractive mappings, Journal of Mathematical Analysis and Applications 332 (2007) 1468-1476.

[12] R. Kannan, Some results on fixed points, Bulletin of Calcutta Mathematical Society 60 (1968) 71-76.

[13] J.J. Nieto, R. Rodríguez-López, Contractive mapping theorems in partially ordered sets and applications to ordinary differential equations, Order 22 (2005) 223-239.

[14] J.J. Nieto, R. Rodríguez-López, Existence and uniqueness of fixed point in partially ordered sets and applications to ordinary differential equations, Acta Mathematica Sinica, English Series 23 (2007) 2205-2212.

[15] J.J. Nieto, R.L. Pouso, R. Rodríguez-López, Fixed point theorems in ordered abstract spaces, Proceedings of the American Mathematical Society 132 (2007) 2505-2517.

[16] D. Paesano, P. Vetro, Suzuki's type characterizations of completeness for partial metric spaces and fixed points for partially ordered metric spaces, Topology and its Applications 159 (2012) 911-920. 
[17] A.C.M. Ran, M.C. Reurings, A fixed point theorem in partially ordered sets and some applications to matrix equations, Proceedings of the American Mathematical Society 132 (2004) 1435-1443.

[18] D. Reem, S. Reich, A.J. Zaslavski, Two Results in Metric Fixed Point Theory, Journal of Fixed Point Theory and Applications 1 (2007) 149-157.

[19] S. Reich, Some remarks concerning contraction mappings, Canadian Mathematical Bulletin 14 (1971) 121-124.

[20] S. Reich, A. J. Zaslavski, A Fixed Point Theorem for Matkowski Contractions, Fixed Point Theory 8 (2007) 303-307.

[21] S. Reich, A.J. Zaslavski, A Note on Rakotch contraction, Fixed Point Theory 9 (2008) 267-273.

[22] I.A. Rus, Generalized Contractions and Applications, Cluj University Press, Cluj-Napoca, 2001.

[23] I.A. Rus, A. Petruşel, G. Petruşel, Fixed Point Theory, Cluj University Press, Cluj-Napoca, 2008.

[24] R. Saadati, S.M. Vaezpour, P. Vetro, B.E. Rhoades, Fixed point theorems in generalized partially ordered G-metric spaces, Mathematical and Computer Modelling 52 (2010) 797-801.

[25] P. Salimi, C. Vetro, P. Vetro, Fixed point theorems for twisted $(\alpha, \beta)-\psi$-contractive type mappings and applications, Filomat 27 (2013) 605-615.

[26] T. Suzuki, A new type of fixed point theorem in metric spaces, Nonlinear Analysis: Theory, Methods \& Applications 71 (2009) 5313-5317.

[27] P. Vetro, Common fixed points in cone metric spaces, Rendiconti del Circolo Matematico di Palermo 56 (2007) 464-468.

[28] D. Wardowski, Fixed points of a new type of contractive mappings in complete metric spaces, Fixed Point Theory and Applications 2012:94 (2012) 